\title{
A Cell-Based Alternative to the Mouse Potency Assay for Pharmaceutical Type E Botulinum Antitoxins
}

\author{
Eran Diamant I\#, Amram Torgeman 1\#, Eyal Epstein'1, Adva Mechaly², Alon Ben David', Lilach Levin'1, \\ Arieh Schwartz', Eyal Dorl, Meni Girshengorn'1, Ada Barnea ${ }^{1}$, Ohad Mazor² and Ran Zichell \\ ${ }^{1}$ Department of Biotechnology, Israel Institute for Biological Research, Ness Ziona, Israel; ${ }^{2}$ Department of Infectious Diseases, Israel Institute for \\ Biological Research, Ness Ziona, Israel
}

\begin{abstract}
The pharmacopeia mouse neutralization assay (PMNA) is the standard method for determining the potency of pharmaceutical botulinum antitoxins. However, a PMNA requires a large number of mice, and, thus, an alternative in vitro method to replace it is needed. Herein, we developed an in vitro SiMa cell line-based neutralization assay (SBNA), compatible with a PMNA design, for therapeutic antitoxins against type E botulinum neurotoxin (BoNT/E). The SBNA measures the residual cellular activity of BoNT/E following antitoxin neutralization in the SiMa lysate using a specific quantitative sandwich ELISA for its cleaved cellular target protein SNAP-25. The potencies of different pharmaceutical antitoxin preparations were determined by applying two different quantification approaches: (1) a cutoff value, in accordance with the pharmacopeia concept, and (2) nonlinear regression of a standard curve generated by serial dilutions of a standard antitoxin. Both approaches achieved accurate potencies compared to the PMNA (average \%RE of 16\%). Furthermore, the SBNA was able to determine in vitro, for the first time, the accurate neutralizing activity $(\% R E \leq 20)$ of next-generation equine and rabbit therapeutic antitoxins. Collectively, a high correlation between SBNA and PMNA results was obtained for all antitoxin preparations $(r=0.99, P<0.0001$ for the standard curve approach, and $r=0.97$, $p<0.0001$ for the cutoff approach). In conclusion, the SBNA can potentially replace the PMNA and markedly reduce the need for laboratory animals for the approval of botulinum antitoxin preparations.
\end{abstract}

\section{Introduction}

Botulinum neurotoxins (BoNTs) are synthesized by the anaerobic bacterium Clostridium botulinum and are the most potent toxins known in nature, with an estimated human median lethal dose $\left(\mathrm{HLD}_{50}\right)$ of $1 \mathrm{ng} / \mathrm{kg}$ of body weight (Arnon et al., 2001). Among the different serotypes of the toxin, A, B, E, and rarely $\mathrm{F}$ have been documented as toxic to humans (Pirazzini et al., 2017). BoNTs exert their toxicity within cholinergic nerve cells by a molecular mechanism of action that includes three steps (Schiavo et al., 2000). First, the C-terminal portion of the toxin heavy chain $\left(\mathrm{H}_{\mathrm{C}}\right)$ binds to receptors on the presynaptic nerve ending membrane. Second, internalization and release of the light chain into the cytosol is facilitated by the translocation domain on the
$\mathrm{N}$-terminus of the heavy chain $\left(\mathrm{H}_{\mathrm{N}}\right)$. Third, one of three soluble N-ethylmaleimide-sensitive factor attachment protein receptor (SNARE) proteins is cleaved by the light chain (LC), a zinc-dependent endopeptidase, thereby preventing the release of the neurotransmitter acetylcholine from nerve cells into synapses (Montal, 2010; Rusnak and Smith, 2009; Smith and Rusnak, 2007). The LC of each BoNT serotype cleaves its protein substrate at a specific site. BoNT/E, the fastest acting of all BoNT serotypes (Wang et al., 2008), cleaves the 25-kDa synaptosomal associated protein (SNAP-25) between Arg180 and Ile181 (Binz et al., 1994; Schiavo et al., 1993).

Standard therapy for botulism in adults includes treatment with equine antitoxin preparations (Dembek et al., 2007; http:// www.emergentbiosolutions.com). According to European Phar-

\# contributed equally

Received May 25, 2021: Accepted November 5, 2021; Epub November 19, 2021; (C) The Authors, 2022

ALTEX 39(1), 113-122. doi:10.14573/altex.2105251

Correspondence: Ran Zichel, PhD IIBR, Ness Ziona 7410001, Israel (ranz@iibr.gov.il)
This is an Open Access article distributed under the terms of the Creative Commons Attribution 4.0 International license (http://creativecommons.org/licenses/by/4.0/) which permits unrestricted use, distribution and reproduction in any medium, provided the original work is appropriately cited. 
macopoeia (PhEur), the potency of these antitoxins is determined by the pharmacopeia mouse neutralization assay (PMNA) (EDQM, 2019a). In this assay, antitoxin potency is determined by injecting mice with mixtures of a fixed toxin dose (a test dose) that has been preincubated with increasing dilutions of the antitoxin preparation to be examined. Neutralizing activity is then determined based on a cutoff approach, in which the transition from mouse mortality to survival between consecutive antitoxin dilutions is used to assess the neutralizing antibody concentration of the antitoxin compared to a reference antitoxin. In addition to the PMNA, the potencies of pharmaceutical BoNT products are routinely tested by the mouse bioassay (MBA), as guided by PhEur (EDQM, 2019b). Altogether, the PMNA and MBA require a high number of laboratory animals (Taylor et al., 2019).

In an effort to implement the $3 \mathrm{R}$ principles (replacement, reduction and refinement) (Tannenbaum and Bennett, 2015), a variety of alternative in vitro methods have been developed to replace the PMNA (Dressler and Dirnberger, 2001; Hanna and Jankovic, 1998; Lindsey et al., 2003; Palace et al., 1998; Rosen et al., 2016) and the MBA (Behrensdorf-Nicol et al., 2018; Wild et al., 2016). Nevertheless, a vast majority of alternative methods are based only on one of the three intoxication steps (binding, internalization, or cleavage) and hence might suffer from poor correlation with the in vivo method.

Alternatively, cell-based assays that include all of the intoxication steps represent the entire intoxication process, resembling the in vivo process, and have the potential to enable an unbiased measurement of toxin potency and antitoxin neutralizing activity. Indeed, several cell-based assays have been developed to measure BoNT activity for pharmaceutical and clinical purposes (Bak et al., 2017; Fernandez-Salas et al., 2012; Hall et al., 2004; Kiris et al., 2014; McNutt et al., 2011; Nuss et al., 2010; Pellett et al., 2011, 2007, 2017, 2010; Rust et al., 2017; Yadirgi et al., 2017; Pathe-Neuschäfer-Rube et al., 2015, 2018; Torgeman et al., 2017a). However, no systematic and direct quantitative comparison to PMNA values of cell-based assays developed to measure the potency of anti-BoNT/E preparations have been described.

The study reported herein is the first to demonstrate concomitant methodical, cell-based measurements and PMNA measurements of pharmaceutical type E botulinum antitoxins with unequivocally high correlation. The developed assay measures residual BoNT/E activity in the human neuroblastoma SiMa cell line (Marini et al., 1999) following neutralization with antitoxin preparations using a specific quantitative sandwich ELISA for cleaved SNAP-25. This in vitro SiMa-based neutralization assay (SBNA) was designed to be consistent with the PMNA by using a calibrated toxin test concentration and a calibrated standard antitoxin (the World Health Organization International Standard (WHO IS) antitoxin), and by applying the cutoff approach (Torgeman et al., 2017a). Additionally, potency in this study was also determined by nonlinear regression from a standard curve. Both approaches enabled the accurate determination of potencies that correlated well with corresponding PMNA values, thus validating the suitability of the SiMa-based neutralization assay. The relevance of the SBNA was demonstrated for equine and rabbit antibody preparations targeting either the toxin complex prepared from Clostridium botulinum supernatant or the recombinantly expressed receptor binding domain of BoNT/E $\left(\mathrm{rH}_{\mathrm{C}} / \mathrm{E}\right)$. The latter is considered a next-generation therapeutic antitoxin approach because the Hc fragment is nontoxic, safely produced, and carries most of the BoNT neutralizing epitopes (Zarebski et al., 2008).

\section{Animals, materials and methods}

\section{Laboratory animals}

Experiments were approved by the IIBR Animal Care and Use Committee, and animals were maintained in accordance with the guidelines of the care and use of laboratory animals published by the Israeli Ministry of Health (protocols RB-27-16, M-77-15, M-65-17, H-02-2014). Animals were acclimated for four days before commencing any procedures. All animals were observed for morbidity and mortality, overt signs of toxicity, and any signs of distress throughout the study. All efforts were made to minimize animal suffering including the use of analgesics and anesthetics. Animals were fed and had access to fresh water ad libitum.

Male and female horses (age from 7 to 15 years, weighing approximately between 450 to $600 \mathrm{~kg}$ ) belonging to the breeds Quarter palomino, Thoroughbred (mixed breed), and Holsteiner (mixed breed) were maintained in the IIBR GMP stable facility and used for the production of hyperimmune plasma. During this procedure, detomidine (up to $30 \mathrm{mg}$, IV) and lidocaine $(2 \%, 0.5 \mathrm{~mL}, \mathrm{SC})$ were given as a sedative and anesthetic respectively.

Female New Zealand White (NZW) rabbits (weighing between 2.5 and $3.5 \mathrm{~kg}$ ) were purchased from Charles River France and used for the production of hyperimmune plasma. In the primary vaccination of rabbits, which included complete Freud's adjuvant, buprenorphine (up to $0.1 \mathrm{mg} / \mathrm{kg}, \mathrm{SC}$ ) was used as an analgesic. For final bleeding, rabbits were given ketamine $(40 \mathrm{mg} / \mathrm{kg}, \mathrm{IM})$ and xylazine $(8 \mathrm{mg} / \mathrm{kg}$, IM) as an anesthetic and sedative, respectively. An endpoint in rabbits was defined as severe infection that cannot be treated with antibiotics.

Female CD-1 mice (weighing between 22 and 27 gr) were purchased from Charles River UK and used in the PMNA and MBA. Mice were not given analgesics and anesthetics. The endpoint in mice was defined as absence of righting reflex.

No animals were excluded from the study.

\section{Toxins and antitoxins}

The Clostridium botulinum E strain was obtained from the IIBR collection (E450). Sequence analysis revealed that the neurotoxin gene was consistent with the serotype of NCTC11219 (GenBank accession number X62683) for Clostridium botulinum subtype E (Whelan et al., 1992). Two batches (TOX1 and TOX2) of the activated form of the BoNT/E complex (consisting of neurotoxin and nontoxic nonhemagglutinin proteins) had been previously prepared and calibrated according to the pharmacopeia. Briefly, a concentrated supernatant of an anaerobic culture grown 
for 6 days was treated with $0.1 \%$ (wt/vol) trypsin $\left(37^{\circ} \mathrm{C}\right.$ for $\left.1 \mathrm{~h}\right)$, and activity was determined by the standard MBA followed by the PMNA. A BoNT/E toxoid was prepared by dialyzing the toxin complex against $0.14 \%$ formalin at $35^{\circ} \mathrm{C}$ for 2 weeks (Diamant et al., 2014). This process detoxifies the antigen while retaining its antigenicity. Recombinant $\mathrm{H}_{\mathrm{C}} / \mathrm{E}\left(\mathrm{rH}_{\mathrm{C}} / \mathrm{E}\right)$ was prepared in E. coli as previously described (Ben David et al., 2013).

Horse anti-BoNT/E antitoxins were collected and purified from two hyperimmune horses immunized against the BoNT/E toxoid as previously described (Diamant et al., 2018; Torgeman et al., 2017b).

Horse anti-rH $\mathrm{C}_{\mathrm{C}} / \mathrm{E}$ antitoxin was collected and purified from one hyperimmune horse immunized against $\mathrm{rH}_{\mathrm{C}} / \mathrm{E}$. The vaccination regimen included three successive subcutaneous (SC) administrations of $10 \mathrm{mg}$ of $\mathrm{rH}_{\mathrm{C}} / \mathrm{E}$ adsorbed to aluminum hydroxide $\left(\mathrm{Al}(\mathrm{OH})_{3}\right)$ [final concentration of $0.5 \%$ (wt/vol)] in intervals of four weeks, followed by one SC administration of $20 \mathrm{mg}$ soluble $\mathrm{rH}_{\mathrm{C}} / \mathrm{E}$ in phosphate buffer.

Equine hyperimmune plasma (HIP) and pharmaceutical drug substance (DS) consisted of whole $\operatorname{IgG}$ and $\left.\mathrm{F}(\mathrm{ab})_{2}\right)_{2}$ purified from horse antitoxin preparations, respectively. The preparations were produced by the method described by Falach et al. (2018) and were provided by the IIBR GMP-approved manufacturing facility of pharmaceutical BoNT antitoxins.

A rabbit anti-BoNT/E antitoxin was collected and purified from three hyperimmune rabbits vaccinated with BoNT/E toxoid. The vaccination regimen included injection of $0.5 \mathrm{mg}$ of toxoid by a single SC administration with complete Freund's adjuvant, followed by two intramuscular (IM) administrations with incomplete Freund's adjuvant and four administrations of soluble toxoid at four-week intervals.

A rabbit anti- $\mathrm{rH}_{\mathrm{C}} / \mathrm{E}$ antitoxin was collected and purified from one hyperimmune rabbit vaccinated with $\mathrm{rH}_{\mathrm{C}} / \mathrm{E}$. The vaccination regimen included a single $\mathrm{SC}$ administration of $100 \mu \mathrm{g} \mathrm{rH} / \mathrm{E}$ with complete Freund's adjuvant, followed by two SC administrations of $100 \mu \mathrm{g} \mathrm{rH}_{\mathrm{C}} / \mathrm{E}$ with incomplete Freund's adjuvant at four-week intervals, two SC administrations of $100 \mu \mathrm{g}$ soluble $\mathrm{rH}_{\mathrm{C}} / \mathrm{E}$ and a final injection of $1 \mathrm{mg}$ soluble $\mathrm{rH}_{\mathrm{C}} / \mathrm{E}$ at three-month intervals.

The neutralizing activity of all antitoxin preparations was determined by the PMNA according to PhEur (EDQM, 2019a), see below. The standard antitoxin (horse anti-BoNT/E antitoxin) used in the PMNA was calibrated against the WHO IS antitoxin (obtained from NIBSC, UK) (Jones et al., 2006).

\section{SiMa-based neutralization assay}

The cellular potency assay consisted of two steps: (1) in vitro neutralization of BoNT/E with antitoxin followed by incubation in a SiMa cell culture and (2) in vitro quantification of cleaved SNAP-25 in the SiMa lysate by a specific ELISA to measure residual BoNT/E activity.

The human neuroblastoma cell line SiMa (ACC 164, Leibniz Institute, DSMZ - German Collection of Microorganisms and Cell Cultures GmbH, Braunschweig, Germany) was cultured in RPMI 1640 (Biological Industries, Israel) medium and split in a ratio of 1:3 every three days using trypsin/EDTA. Passages 10-20 were used for experiments. Mycoplasma testing was performed by an external certified laboratory (Hylabs, Israel).

$5 \times 10^{4}$ cells per well were seeded in poly-D-lysine-coated 96-well plates (Corning, USA) in differentiation medium containing serum-free Minimum Essential Medium (MEM) with Earl's salts and Glutamax (Gibco) supplemented with N-2 (x1) (Gibco), B-27 (x1) (Gibco), and $25 \mu \mathrm{g} / \mathrm{mL}$ GT1b (Enzo), as previously described (Fernandez-Salas et al., 2012) and allowed to differentiate for two days.

Next, serial dilutions of toxin (TOX1 and TOX2) or toxin-antitoxin mixtures were prepared in GT1b-free differentiation medium. According to the WHO standardization, $1 \mathrm{IU}$ of anti-BoNT/E antitoxin neutralizes at least $10^{3} \mathrm{MsLD}_{50}$ of toxin (Bowmer, 1963). A toxin test concentration (TOX2, $830 \mathrm{MsLD}_{50} / \mathrm{mL}$ ), previously calibrated using the PMNA, was incubated for $1 \mathrm{~h}$ at $25^{\circ} \mathrm{C}$ with 0.01-0.08 international units (IU) per mL of standard antitoxin or with dilutions of test antitoxins.

After differentiation, the medium was removed from the cells and replaced with $100 \mu \mathrm{L}$ fresh, GT1b-free differentiation medium containing either only toxin or toxin-antitoxin mixtures. The experiments were performed with 4 technical replicates. The number of biological replicates is shown in the respective figures. Following incubation for $24 \mathrm{~h}$ at $37^{\circ} \mathrm{C}$, the medium was replaced with fresh MEM, and the cells were incubated for an additional $24 \mathrm{~h}$ at $37^{\circ} \mathrm{C}$.

Cells were then lysed by incubation with a cold solution containing $0.1 \%$ Triton X-100, $150 \mathrm{mM} \mathrm{NaCl}, 1.5 \mathrm{mM} \mathrm{MgCl}_{2}, 1 \mathrm{mM}$ EGTA, $50 \mathrm{mM}$ HEPES, and protease inhibitor cocktail (EDTAfree cOmplete tablets, Roche Diagnostics $\mathrm{GmbH}$, Mannheim, Germany) in water for 30 minutes. Lysates were analyzed for BoNT/E-cleaved SNAP-25 using a specific ELISA.

ELISA was performed in 96-well plates (Maxisorp, Nunclon) precoated with $100 \mathrm{ng} /$ well of a recombinant rabbit-human chimera monoclonal antibody (MAb) that specifically recognizes BoNT/E-cleaved SNAP-25 (Mechaly et al., 2021). This antibody was prepared by immunizing NZW rabbits with a KLH-conjugated peptide comprising eight amino acids (Thr173 - Arg180) of the SNAP-25 sequence preceding the BoNT/E cleavage site (between Arg180 and Ile181) and generating MAbs from lymphatic organ-derived B cells in a phage display expression system (Mechaly et al., 2018, 2019). Plates were blocked with TSTA buffer $(2 \%(\mathrm{w} / \mathrm{v})$ bovine serum albumin (BSA), $0.9 \% \mathrm{NaCl}$, $0.05 \%$ Tween 20 in $50 \mathrm{mM}$ Tris (pH 7.6)). Following blocking and washing, plates were incubated with cell lysates $(50 \mu \mathrm{L})$ for $1 \mathrm{~h}$ at $37^{\circ} \mathrm{C}$. After an additional wash, plates were incubated for $1 \mathrm{~h}$ at $37^{\circ} \mathrm{C}$ with polyclonal rabbit anti-human SNAP-25 (Sigma, MO, USA) diluted 1:4000 in TSTA buffer supplemented with $1 \%$ naïve human serum. This antibody, targeting a peptide located far upstream of the cleavage site, recognizes both cleaved and intact SNAP-25. Plates were washed and then incubated with HRP-conjugated donkey anti-rabbit (Jackson ImmunoResearch Laboratories Inc., PA, USA) diluted 1:4000. The colorimetric reaction was developed using SureBlue substrate (Sera Care, MA, USA) and stopped with $1 \mathrm{~N} \mathrm{H}_{2} \mathrm{SO}_{4}$. Absorbance was measured at $450 \mathrm{~nm}$ with a Synergy HTX multimode reader (BioTek Instruments, Inc., USA). 
In the cutoff approach, potency results were calculated by multiplying the cutoff dilution of the examined antitoxin preparation by the cutoff value of the standard antitoxin. The cutoff dilution was defined similarly to the cutoff value of the standard antitoxin, i.e., the lowest concentration that prevented SNAP-25 cleavage (a residual toxin activity lower than 10\% relative to the toxin signal). In the standard curve approach, potency results were calculated by multiplying the dilution of the examined antitoxin preparation by the fitted standard antitoxin value, based on nonlinear regression of the curve using a 4-parameter logistic fit.

\section{Pharmacopeia mouse neutralization assay (PMNA)}

A validated PMNA was conducted according to PhEur (EDQM, 2019a) to determine the potency of all of the antitoxin preparations, as previously described (Torgeman et al., 2018). Mice were randomly divided into the experimental groups. Seven serial 1.2-fold dilutions of each antitoxin preparation were prepared. Concomitantly, a standard antitoxin preparation (calibrated against the WHO IS antitoxin) was diluted to final concentrations of $0.08,0.10,0.12$ and $0.14 \mathrm{IU} / \mathrm{mL}$. All antitoxin dilutions were then mixed with a fixed toxin test concentration (TOX2, $830 \mathrm{MsLD}_{50} / \mathrm{mL}$ ), and the mixtures were incubated for $1 \mathrm{~h}$ at $25^{\circ} \mathrm{C}$. Each mixture was injected intraperitoneally into four female mice ( $1 \mathrm{~mL}$ per mouse) per group, and survival was monitored for 4 days.

\section{Results}

\subsection{Study design}

The goal of the study was to develop a cell-based in vitro assay to determine the concentration of neutralizing antibodies against $\mathrm{BoNT} / \mathrm{E}$ in antitoxin preparations. The assay is based on measuring the residual cellular activity of the neutralized toxin using a quantitative sandwich ELISA that specifically detects its cleaved cellular target protein SNAP-25 in the lysates of neuroblastoma cells. The experimental design of the SBNA consisted of a fixed toxin test concentration identical to that used in the PMNA, which is preincubated with two sets of antitoxins: 1) different concentrations of a standard antitoxin calibrated to the WHO IS antitoxin or 2) different dilutions of the antitoxin sample. Then, the toxin-antitoxin mixtures are incubated with differentiated SiMa cells, and the BoNT/E-cleaved SNAP-25 concentration in the cell lysates is determined by ELISA.

\subsection{Concentration-response relationships in BoNT/E-treated SiMa cells}

To establish the SBNA, it was essential to first demonstrate a concentration-response relationship between BoNT/E concentrations and measured BoNT/E-cleaved SNAP-25. This was assessed by measuring toxin activity in the cells in the absence of antitoxins (Fig. 1). To this end, SiMa cells were incubated separately with a series of concentrations $\left(250-4000 \mathrm{MsLD}_{50} / \mathrm{mL}\right)$ of two BoNT/E test concentrations (TOX1 and TOX2) which originated from two different batches (preparations) of toxin and had

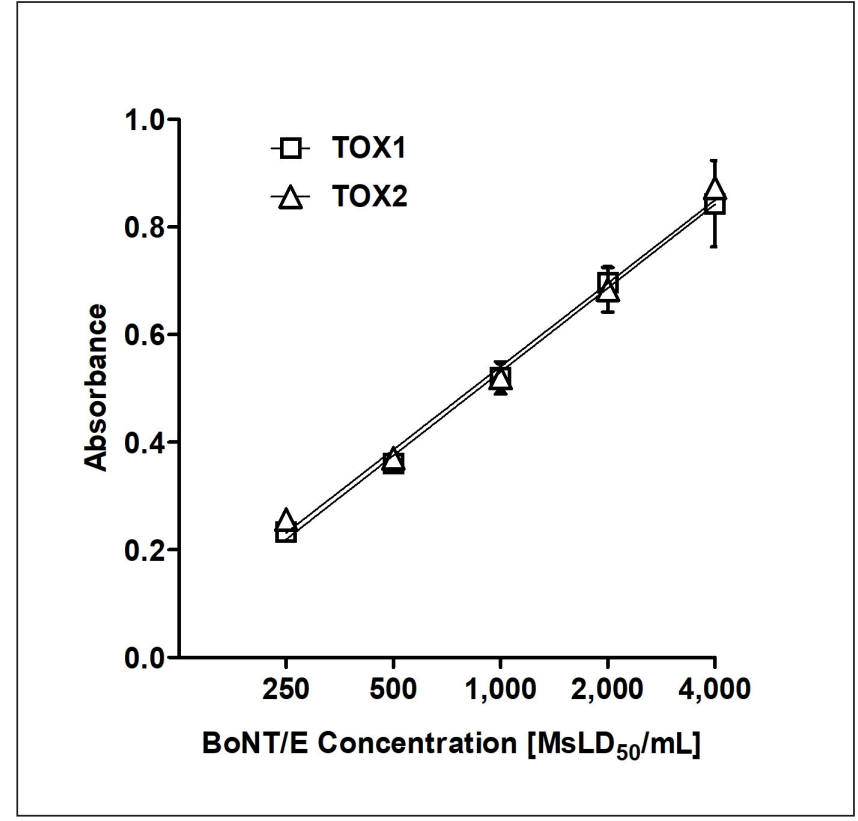

Fig. 1: In vitro SiMa-based activity of BoNT/E

SiMa cells were incubated with two batches of BoNT/E (TOX1 and TOX2) at the indicated concentrations. Toxin activity was determined by a specific ELISA that measured BoNT/E-cleaved SNAP-25 in the cell lysates and is shown as absorbance values. Each data point is the mean $\pm S D$ of $n=4$ technical replicates. Regression analysis demonstrated a goodness of fit of $R^{2}=0.975$ and $R^{2}=0.983$ for TOX1 and TOX2, respectively.

previously been calibrated using the PMNA. Both toxin batches show an almost identical concentration-dependent increase in the level of cleaved SNAP-25 (TOX2 / TOX1 absorbance ratio of $0.98-1.10$ ), with a goodness of fit of $\mathrm{R}^{2}=0.975$ and $\mathrm{R}^{2}=0.983$ for TOX1 and TOX2, respectively. Both toxin batches therefore were suitable as test toxins in the SBNA. This demonstrates that the assay is able to distinguish between toxin concentrations in a proportional manner.

\subsection{Standardization of the cellular assay for quantification of neutralizing antibodies}

We next characterized two approaches that can be applied for potency calculation based on a calibrated standard antitoxin. The first approach adopted the cutoff concept of the PMNA, and the second approach relied on a standard curve.

The cutoff approach was based on the PMNA. Analogous to mouse survival in the PMNA, the cutoff was defined as the lowest antitoxin concentration (i.e., the highest dilution) that prevented SNAP-25 cleavage, resulting in a residual toxin activity that was lower than $10 \%$ relative to the toxin signal in the absence of antitoxin. Figure 2 shows that the BoNT/E standard antitoxin yielded a cutoff value of 0.06 or $0.08 \mathrm{IU} / \mathrm{mL}$ in four independent experiments.

The cell-based assay allows the use of a broad range of serial dilutions of the standard antitoxin, as only small volumes are re- 
A

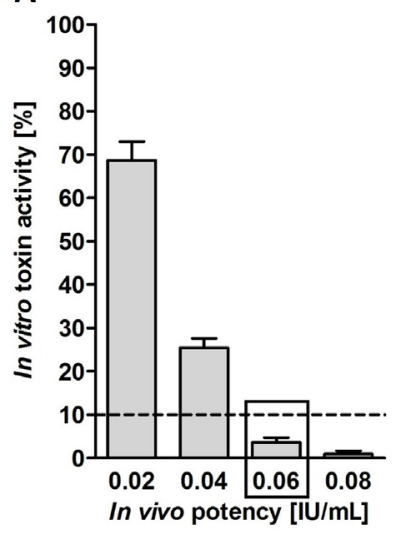

B

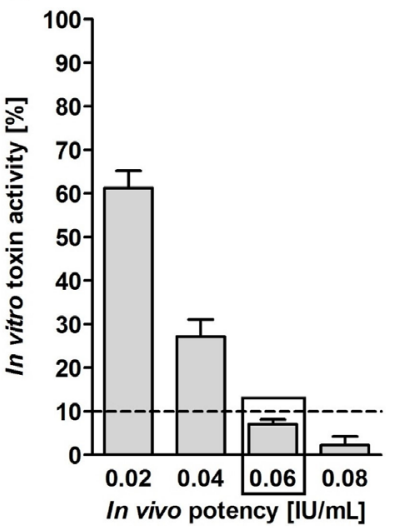

C

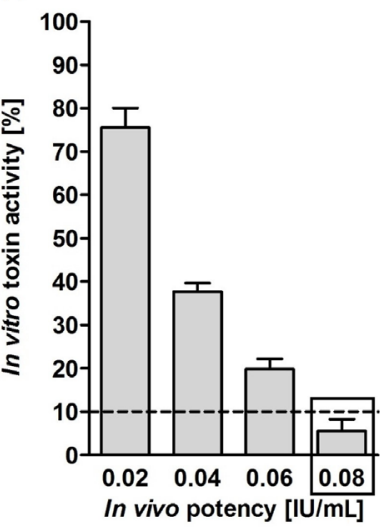

D

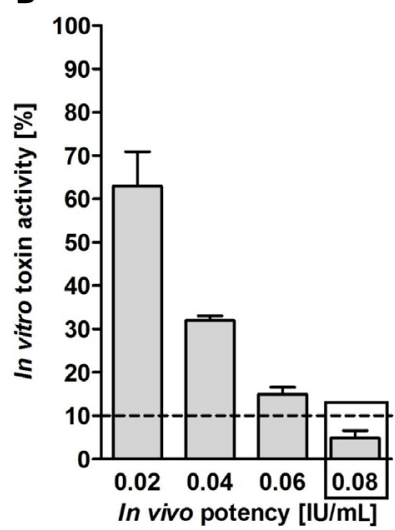

Fig. 2: Determination of antitoxin potency using the SBNA with the cutoff approach

Different concentrations $(0.02,0.04 .0 .06$, and $0.08 \mathrm{IU} / \mathrm{mL})$ of an anti-BoNT/E antitoxin WHO reference standard were incubated with a fixed toxin test concentration (TOX2, $830 \mathrm{MsLD}_{50} / \mathrm{mL}$ ). The cutoff value was determined according to the lowest reference standard antitoxin concentration (the bar enclosed by a rectangle) that prevented SNAP- 25 cleavage (resulting in $\leq 10 \%$ residual activity relative to the toxin signal; dashed line). In all cases, the cutoff value was significantly different compared to the lowest antitoxin concentration above $10 \%$ residual activity ( $p$-value $<0.001$ ). Panels $A, B, C$, and $D$ represent means $\pm S D$ s of four experimental repeats $(N=4, n \geq 3)$.

quired, and animal numbers do not need to be considered as in the PMNA. We used standard antitoxin dilutions from 0.01 to $0.08 \mathrm{IU} / \mathrm{mL}$ to generate a standard curve (Fig. 3). The goodness of fit $\left(\mathrm{R}^{2} \geq 0.96\right)$ suggests that the standard curve approach could be applied for calculation of the neutralizing potency of an unknown antitoxin sample.

\subsection{Evaluation of the in vitro assay performance for potency determination}

Next, the validity of both calculation approaches used to measure antitoxin potency was evaluated. For the in vitro cellular potency assay to serve as a relevant alternative to the in vivo method, it needs to provide potency values of antitoxins that are comparable to those determined using the PMNA. An evaluation of SBNA performance was conducted by comparing in vivo to in vitro potency measurements of BoNT/E antitoxin preparations. The tested preparations included three batches of an IgG equine HIP and three batches of an F(ab') 2 pharmaceutical antitoxin DS (Torgeman et al., 2017b). The neutralizing antibody concentration of the samples was determined by both the cutoff and the standard curve approaches (Fig. 4). The reproducibility of the in vitro assay among all data was high, with a coefficient of variation $(\% \mathrm{CV})$ of $0-10$ for the cutoff and 4.5-8.6 for the standard curve approach.

The two SBNA quantification methods for the HIP and DS samples yielded accurate in vitro potencies with average relative errors $(\% \mathrm{RE})$ of $16 \%$ and $14 \%$ for the cutoff and the standard

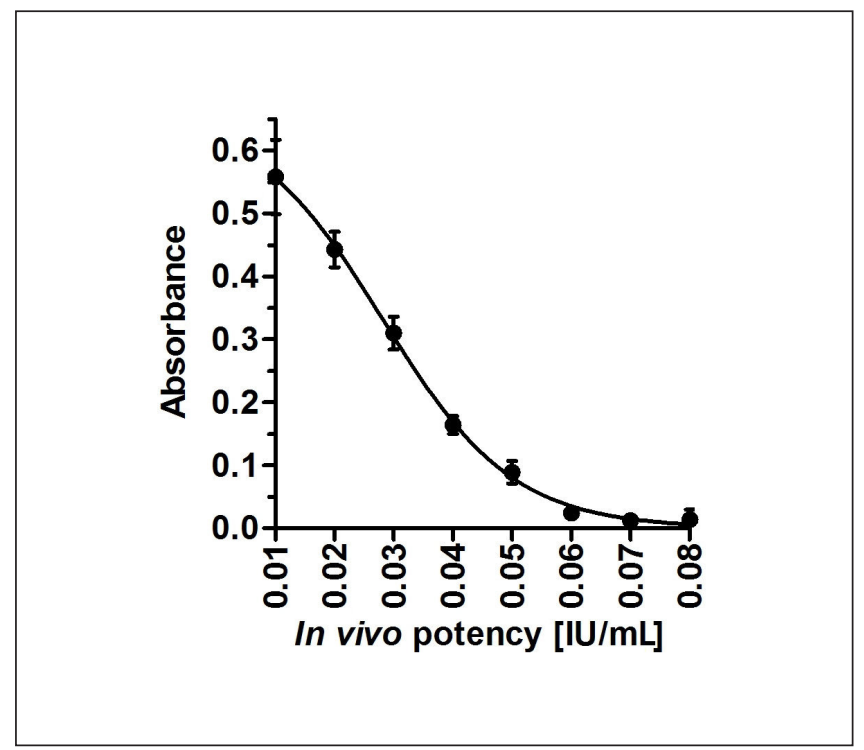

Fig. 3: Standard curve used to determine antitoxin potency using the SBNA

Increasing concentrations ( 0.01 to $0.08 \mathrm{IU} / \mathrm{mL}$ ) of an anti-BoNT/E antitoxin WHO reference standard were incubated with a fixed toxin test concentration (TOX2, $830 \mathrm{MsLD}_{50} / \mathrm{mL}$ ). Standard curves for the residual BoNT/E activity were plotted using a 4-parameter logistic curve fit. A representative curve is shown. Data points are means $\pm S D(n \geq 3)$. 


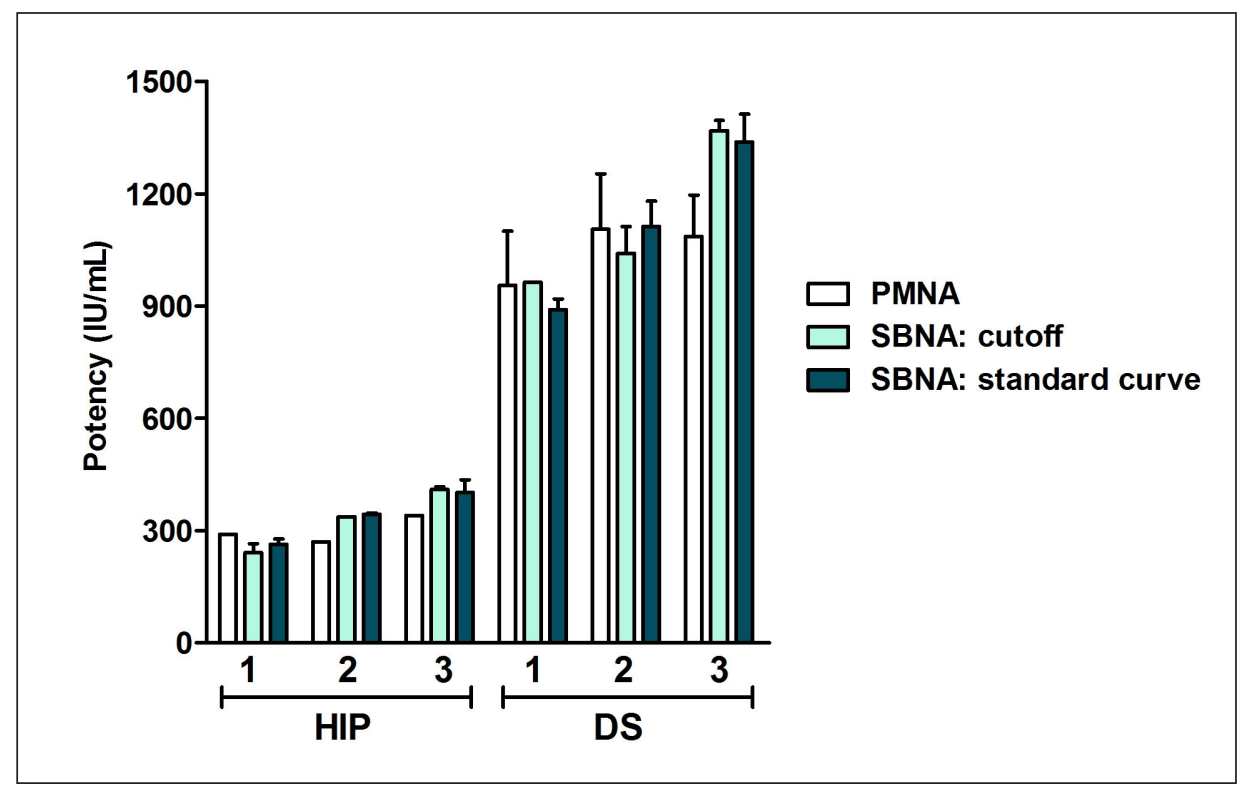

Fig. 4: In vitro determination of antitoxin test batch potency in comparison to the PMNA

The potencies of three batches each of equine HIP and pharmaceutical antitoxin DS (designated 1, 2, and 3) were calculated by applying either the pharmacopeia cutoff or the standard curve approach and compared to the PMNA value. The PMNA values for HIP were determined in a single experiment $(N=1)$; values for DS samples were from three experiments as part of the requirements to license the product as a pharmaceutical drug $(\mathrm{N}=3)$. The in vitro data were determined in 1 or 2 experiments, where each data set was the mean $\pm S D$ of $n=4$ replicates.

Tab. 1: In vivo and in vitro potencies of rabbit and next-generation equine antitoxins

\begin{tabular}{|c|c|c|c|c|c|}
\hline \multirow[t]{3}{*}{ Antitoxin preparation } & \multirow[t]{3}{*}{ PMNA $^{a}(\mathrm{IU} / \mathrm{mL})$} & \multicolumn{4}{|l|}{ SBNA $^{b}$} \\
\hline & & \multicolumn{2}{|l|}{ Standard curve } & \multicolumn{2}{|l|}{ Cutoff } \\
\hline & & Potency (IU/mL) & $\%$ RE & Potency (IU/mL) & $\% R E$ \\
\hline Horse anti-rH $\mathrm{C} / \mathrm{E}$ fragment & 625 & 697 & 12 & 723 & 16 \\
\hline Rabbit anti-BoNT/E complex & 125 & 134 & 10 & 115 & 4 \\
\hline Rabbit anti $\mathrm{rH}_{\mathrm{C}} / \mathrm{E}$ fragment & 183 & 205 & 11 & 231 & 20 \\
\hline
\end{tabular}

${ }^{a}$ In vivo potency values were determined by the validated PMNA (seven antitoxin dilutions per sample, four mice per dilution). ${ }^{\mathrm{b}}$ In vitro results represent the average of triplicates ( $n=$ three wells per dilution).

curve approach, respectively, compared to the PMNA results, which is in line with the acceptance criteria for ligand binding assays (DeSilva et al., 2003). Thus, these data clearly demonstrate that both SBNA determination methods result in potency values that are comparable to those obtained by the PMNA.

\subsection{Evaluation of SBNA performance in rabbit and next-generation equine antitoxins}

Current standard drug therapy for botulism in adult patients relies on equine antibody preparations targeting the toxin complex. Some efforts to develop next-generation antitoxins focus on vaccination of horses with the neurotoxin receptor binding domain (BoNT $\mathrm{H}_{\mathrm{C}}$ fragment) rather than with the toxoid of either the BoNT holotoxin or complex (Ben David et al., 2013, 2015).

Therefore, we measured the potency of plasma preparations derived from $\mathrm{rH}_{\mathrm{C}} / \mathrm{E}$ vaccinated horses using PMNA and SBNA approaches and compared the results. In addition to equine antitoxins, rabbit preparations were tested. These antibodies were generated in rabbits against the BoNT/E complex or specifically against the $\mathrm{rH}_{\mathrm{C}} / \mathrm{E}$ fragment. Collectively, the neutralizing activity results obtained using the SBNA, either by the standard curve approach or by the cutoff approach, were comparable to their respective in vivo potencies, with an acceptable low relative error (\%RE) of less than 20\% (Tab. 1) (DeSilva et al., 2003).

\subsection{Overall correlation of SBNA with PMNA results}

To assess the relationship between SBNA and PMNA results, statistical analyses were conducted to calculate the overall correlation and to compare the means. Altogether, neutralizing antibody potency results for the HIP, DS batches, rabbit anti-BoNT/E complex, and for anti- $\mathrm{rH}_{\mathrm{C}} / \mathrm{E}$ from both horses and rabbits revealed accurate potencies with $\% \mathrm{RE} \leq 20$ on average for a total of nine antitoxin preparations for both quantification approaches.

The mean neutralizing antibody potencies of the nine antitoxin preparations were compared with two-tailed paired $t$-tests. There were no significant differences between the PMNA and the SBNA ( $p$-value $=0.18$ and $p$-value $=0.17$ for the cutoff and the standard curve approaches, respectively). These data support the 
A

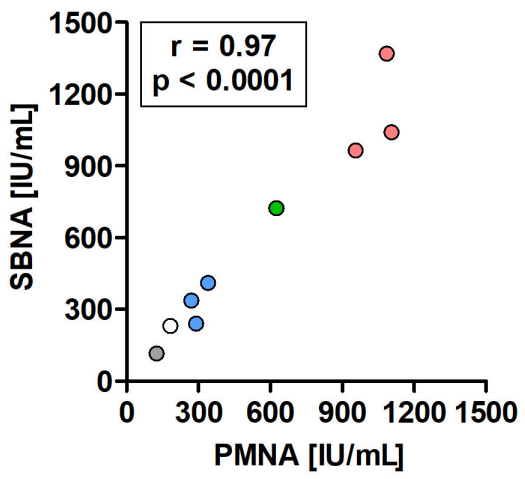

B

Standard curve

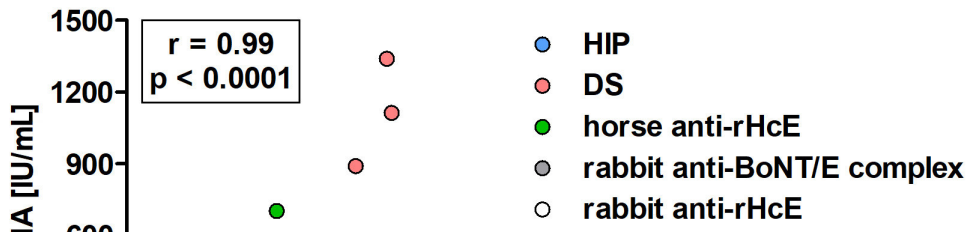

Fig. 5: Overall correlation between antitoxin potency determined by the in vitro assay and the PMNA in various BoNT/E antitoxin preparations

Samples include three batches of HIP, three batches of an $\left.\mathrm{F}(\mathrm{ab})_{2}\right)_{2}$ pharmaceutical antitoxin $\mathrm{DS}$, one batch of horse anti-rH $\mathrm{H}_{\mathrm{C}} / \mathrm{E}$, one batch of rabbit anti-BoNT/E complex antitoxin, and one batch of rabbit anti-rH $\mathrm{H}_{\mathrm{C}} / \mathrm{E}$ preparation. The in vitro potency was obtained by applying the cutoff (panel A) and the standard curve (panel B) approaches.

$\%$ RE results, indicating similar potencies were obtained using both SBNA approaches compared to the PMNA.

In addition, the neutralization results demonstrate a high correlation between the PMNA and the SBNA: $r=0.97$ (confidence interval $(\mathrm{CI})=0.878-0.995)$ and $\mathrm{p}<0.0001$ for the cutoff approach and $r=0.99(\mathrm{CI}=0.902-0.996)$ and $\mathrm{p}<0.0001$ for the standard curve approach (Fig. 5). Collectively, these data confirm that the SBNA allows accurate determination of the potency of a variety of antitoxin preparations.

\section{Discussion}

Therapy with antitoxins for either BoNT/A, BoNT/B, or $\mathrm{BoNT} / \mathrm{E}$ is relevant for the vast majority of human botulism cases. The PMNA is currently the only approved method to determine the potency of pharmaceutical botulinum antitoxin preparations (EDQM, 2019a). The exact number of mice used worldwide to test therapeutic BoNT antitoxin preparations is not known but is considered to be high. In addition to the use of mice for the PMNA, it is estimated that hundreds of thousands of mice are used worldwide per year to test pharmaceutical BoNT batches in the MBA, although proprietary in vitro assays are in use by BoNT producers such as Allergan, Ipsen, and Merz (Bitz, 2010; Taylor et al., 2019). Altogether, there is a serious need to develop alternative in vitro assays to replace the in vivo methods or at least to reduce the number of mice required to determine the potency of BoNTs and respective antitoxins in the pharmaceutical industry.
As part of the effort to implement the $3 \mathrm{R}$ principles, a recent study from our lab presented the development of a SBNA to determine BoNT/A antitoxin potency (Torgeman et al., 2017a). As SiMa cells present low sensitivity to BoNT/B intoxication, we developed an in vitro cell-free endopeptidase-based neutralization assay for determining BoNT/B antitoxin potency (Rosen et al., 2016). Later, Rust et al. re-engineered the VAMP molecule in SiMa cells to allow sensitive detection of BoNT/B intoxication (Rust et al., 2017), but this method has not been further applied to test the potency of BoNT/B antitoxin. To complete the in vitro alternative toolkit for replacing the PMNA in assessing the potency of antitoxins for human botulism, the present study establishes a SiMa cell-based neutralization assay for BoNT/E antitoxins. This method was further shown to be suitable for the evaluation of next-generation equine and rabbit antitoxin preparations.

A prerequisite to establishing such an in vitro assay is the ability to accurately detect differences in BoNT/E-cleaved SNAP-25 values derived from residual BoNT/E concentrations in the presence of anti-BoNT/E antitoxin. Accordingly, we first demonstrated a precise and almost identical concentration-dependent response to two batches of toxin, originally calibrated as test doses for the PMNA (ranging from 250 to $4000 \mathrm{MsLD}_{50} / \mathrm{mL}$ ), in the absence of antitoxin preparations. In addition to measuring the potency of antitoxin preparations, the SBNA can therefore be used directly for in vitro determination of $\mathrm{LD}_{50}$ potency in toxin samples and thereby is suitable to replace the MBA.

Two important principles were applied in the assay design to fully comply with the PhEur concepts adopted for the PMNA 
(EDQM, 2019a). First, a PMNA-calibrated BoNT/E test concentration and a standard antitoxin calibrated to the WHO IS antitoxin were utilized. Second, similar to the pharmacopeia method, a cutoff approach was applied to determine the in vitro potency of the tested samples. Notably, we previously demonstrated the validity of this concept using an in vitro cell-based potency assay for therapeutic BoNT/A-equine antitoxins (Torgeman et al., 2017a). Yet, according to the guidelines of the U.S. Food and Drug Administration (FDA) and the International Council for Harmonisation ( $\mathrm{ICH}$ ), a calibration curve should be used during the development of a bioassay (ICH, 2019; US FDA, 2018). Therefore, in addition to the PMNA cutoff concept, we were able to implement a standard curve approach in which potency quantification was based on a continuous value range instead of a discrete value as in the cutoff approach. In this regard, a standard curve approach can more precisely determine the antitoxin potency than arbitrary discrete dilutions of the standard antitoxin in the cutoff approach can. The standard curve approach is feasible in the SBNA as the amount of antitoxin required is low and animal numbers do not need to be considered as in the PMNA. Importantly, similar results were obtained using both methods.

The cutoff value in the newly developed SBNA was found to be either 0.06 or $0.08 \mathrm{IU} / \mathrm{mL}$ in four independent experiments. Similarly, the cutoff in the PMNA is also based on more than one possible value (either 0.08 0.1, or $0.12 \mathrm{IU} / \mathrm{mL}$ ) (EDQM, 2019a). It should be noted that the PMNA $(0.08-0.12 \mathrm{IU} / \mathrm{mL})$ and the SBNA (0.06-0.08 IU/mL) were highly correlated. The difference in the cutoff values may be related to the different measured endpoints of the assays. While the SBNA measures the in vitro catalytic outcome, the PMNA measures the mortality or survival of mice. A similar finding was observed in our previous study, which showed that the SBNA for pharmaceutical BoNT/A antitoxins also had a cutoff value $(0.04 \mathrm{IU} / \mathrm{mL})$ that differed from that of the PMNA (0.08-0.12 IU/mL) (Torgeman et al., 2017a).

The assay validity was confirmed by a direct comparison between SBNA and PMNA potencies obtained for various preparations, including equine and rabbit antitoxins targeting either the whole BoNT/E or $\mathrm{rH}_{\mathrm{C}} / \mathrm{E}$. The potencies of these antitoxin preparations were accurately determined by both the standard curve and the cutoff quantification methods. The mean neutralizing antibody potencies of the nine antitoxin preparations obtained using both SBNA approaches were not significantly different from their PMNA results, indicating similar potencies. Furthermore, the in vitro results were highly reproducible, as the precision was found to be high $(\% \mathrm{CV}<10)$. In addition, correlations between PMNA and SBNA potencies were highly significant. The fact that preparations from different species (horse and rabbit) and preparations generated against different antigens (complex BoNT/E and subunit $\mathrm{rH}_{\mathrm{C}} / \mathrm{E}$ ) were all determined accurately further validates both SBNA analysis approaches.

Several previous studies have used cell-based assays for in vitro quantification of neutralizing antibodies (Bak et al., 2017; Hall et al., 2004; Nuss et al., 2010; Pellett et al., 2007; Yadirgi et al., 2017). However, the vast majority of the in vitro values were not compared to respective PMNA data. To date, only one cell-based study has attempted to assess the in vitro neutraliz- ing activity of type $\mathrm{E}$ botulinum antitoxins with respect to the PMNA, and its evaluation was limited since the in vivo potency was not precisely defined but was based on an assumed broad range ( $>50 \mathrm{IU} / \mathrm{mL}$, stated by the antitoxin manufacturer) (Bak et al., 2017).

Owing to our ability to carry out in vivo (PMNA) and in vitro (SBNA) assays in parallel and to compare antitoxin preparation potencies directly, our study with $\mathrm{BoNT} / \mathrm{E}$ is the first to accurately validate its results by comparing them to respective PMNA values. Furthermore, this in vitro assay is the first to accurately measure the potency of next-generation antitoxin preparations from equine and rabbit sources. The data support the suitability of the SBNA as an in vitro alternative that can replace the PMNA in determining the potencies of a wide range of botulinum antitoxin products and thereby can dramatically reduce the use of laboratory animals for this purpose.

\section{References}

Arnon, S. S., Schechter, R., Inglesby, T. V. et al. (2001). Botulinum toxin as a biological weapon: Medical and public health management. JAMA 285, 1059-1070. doi:10.1001/jama. 285.8.1059

Bak, N., Rajagopal, S., Stickings, P. et al. (2017). SiMa cells for a serotype specific and sensitive cell-based neutralization test for botulinum toxin A and E. Toxins (Basel) 9, 230. doi:10.3390/ toxins 9070230

Behrensdorf-Nicol, H. A., Wild, E., Bonifas, U. et al. (2018). In vitro potency determination of botulinum neurotoxin serotype A based on its receptor-binding and proteolytic characteristics. Toxicol In Vitro 53, 80-88. doi:10.1016/j.tiv.2018.07.008

Ben David, A., Diamant, E., Barnea, A. et al. (2013). The receptor binding domain of botulinum neurotoxin serotype A (BoNT/ A) inhibits BoNT/A and BoNT/E intoxications in vivo. Clin Vaccine Immunol 20, 1266-1273. doi:10.1128/cvi.00268-13

Ben David, A., Torgeman, A., Barnea, A. et al. (2015). Expression, purification and characterization of the receptor-binding domain of botulinum neurotoxin serotype B as a vaccine candidate. Protein Expr Purif 110, 122-129. doi:10.1016/j. pep.2015.02.008

Binz, T., Blasi, J., Yamasaki, S. et al. (1994). Proteolysis of SNAP-25 by types E and A botulinal neurotoxins. J Biol Chem 269, 1617-1620. doi:10.1016/S0021-9258(17)42071-0

Bitz, S. (2010). The botulinum neurotoxin LD $_{50}$ test - Problems and solutions. ALTEX 27, 114-116. doi:10.14573/altex. 2010.2.114

Bowmer, E. J. (1963). Preparation and assay of the international standards for Clostridium botulinum types A, B, C, D and E antitoxins. Bull World Health Organ 29, 701-709.

Dembek, Z. F., Smith, L. A. and Rusnak, J. M. (2007). Botulism: Cause, effects, diagnosis, clinical and laboratory identification, and treatment modalities. Disaster Med Public Health Prep 1, 122-134. doi:10.1097/DMP.0b013e318158c5fd

DeSilva, B., Smith, W., Weiner, R. et al. (2003). Recommendations for the bioanalytical method validation of ligand-binding assays to support pharmacokinetic assessments of macro- 
molecules. Pharm Res 20, 1885-1900. doi:10.1023/b:pham. $0000003390.51761 .3 \mathrm{~d}$

Diamant, E., Lachmi, B. E., Keren, A. et al. (2014). Evaluating the synergistic neutralizing effect of anti-botulinum oligoclonal antibody preparations. PLoS One 9, e87089. doi:10.1371/ journal.pone.0087089

Diamant, E., Pass, A., Rosen, O. et al. (2018). A novel rabbit spirometry model of type E botulism and its use for the evaluation of postsymptom antitoxin efficacy. Antimicrob Agents Chemother 62, e02379-17. doi:10.1128/AAC.02379-17

Dressler, D. and Dirnberger, G. (2001). Botulinum toxin antibody testing: Comparison between the immunoprecipitation assay and the mouse diaphragm assay. Eur Neurol 45, 257260. doi: $10.1159 / 000052139$

EDQM - European Directorate for the Quality of Medicines and Healthcare (2019a). Botulinum Toxin Type A / Type B for Injection. European directorate for the quality of medicines and healthcare. Vol. 10. Strasbourg, France: European Pharmacopoeia.

EDQM (2019b). Botulinum Antitoxin. European directorate for the quality of medicines and healthcare. Vol. 10. Strasbourg, France: European Pharmacopoeia.

Falach, R., Sapoznikov, A., Alcalay, R. et al. (2018). Generation of highly efficient equine-derived antibodies for post-exposure treatment of ricin intoxications by vaccination with monomerized ricin. Toxins (Basel) 10, 466. doi:10.3390/toxins 10110466

Fernandez-Salas, E., Wang, J., Molina, Y. et al. (2012). Botulinum neurotoxin serotype A specific cell-based potency assay to replace the mouse bioassay. PLoS One 7, e49516. doi:10.1371/journal.pone.0049516

Hall, Y. H., Chaddock, J. A., Moulsdale, H. J. et al. (2004). Novel application of an in vitro technique to the detection and quantification of botulinum neurotoxin antibodies. J Immunol Methods 288, 55-60. doi:10.1016/j.jim.2004.02.011

Hanna, P. A. and Jankovic, J. (1998). Mouse bioassay versus western blot assay for botulinum toxin antibodies: Correlation with clinical response. Neurology 50, 1624-1629. doi:10.1212/ WNL.50.6.1624

ICH - International Council for Harmonisation (2019). ICH Guideline M10 on Bioanalytical Method Validation. https:// www.Ema.Europa.Eu/en/documents/scientific-guideline/ draft-ich-guideline-m10-bioanalytical-method-validationstep-2b_en.pdf

Jones, R. G., Corbel, M. J. and Sesardic, D. (2006). A review of WHO international standards for botulinum antitoxins. Biologicals 34, 223-226. doi:10.1016/j.biologicals.2005.11.009

Kiris, E., Kota, K. P., Burnett, J. C. et al. (2014). Recent developments in cell-based assays and stem cell technologies for botulinum neurotoxin research and drug discovery. Expert Rev Mol Diagn 14, 153-168. doi:10.1586/14737159.2014.867808

Lindsey, C. Y., Smith, L. A., West, M. W. et al. (2003). Evaluation of a botulinum fragment C-based ELISA for measuring the humoral immune response in primates. Biologicals 31, 1724. doi:10.1016/S1045-1056(02)00074-X

Marini, P., MacLeod, R. A., Treuner, C. et al. (1999). SiMa, a new neuroblastoma cell line combining poor prognostic cytogenetic markers with high adrenergic differentiation. Cancer Genet Cytogenet 112, 161-164. doi:10.1016/s01654608(98)00269-6

McNutt, P., Celver, J., Hamilton, T. et al. (2011). Embryonic stem cell-derived neurons are a novel, highly sensitive tissue culture platform for botulinum research. Biochem Biophys Res Commun 405, 85-90. doi:10.1016/j.bbrc.2010.12.132

Mechaly, A., Alcalay, R., Noy-Porat, T. et al. (2018). Novel phage display-derived anti-abrin antibodies confer post-exposure protection against abrin intoxication. Toxins (Basel) 10, 80. doi:10.3390/toxins 10020080

Mechaly, A., Elia, U., Alcalay, R. et al. (2019). Inhibition of francisella tularensis phagocytosis using a novel anti-LPS scFv antibody fragment. Sci Rep 9, 11418. doi:10.1038/s41598-01947931-w

Mechaly, A., Diamant, E., Alcalay, R. et al. (2021). Isolation and characterization of a highly specific monoclonal antibody targeting the botulinum neurotoxin type E exposed SNAP-25 neoepitope. bioRxiv. doi:10.1101/2021.09.16.460610

Montal, M. (2010). Botulinum neurotoxin: A marvel of protein design. Ann Rev Biochem 79, 591-617. doi:10.1146/annurev. biochem.051908.125345

Nuss, J. E., Ruthel, G., Tressler, L. E. et al. (2010). Development of cell-based assays to measure botulinum neurotoxin serotype A activity using cleavage-sensitive antibodies. J Biomol Screen 15, 42-51. doi:10.1177/1087057109354779

Palace, J., Nairne, A., Hyman, N. et al. (1998). A radioimmunoprecipitation assay for antibodies to botulinum A. Neurology 50, 1463-1466. doi:10.1212/WNL.50.5.1463

Pathe-Neuschäfer-Rube, A., Neuschäfer-Rube, F., Genz, L. et al. (2015). Botulinum neurotoxin dose-dependently inhibits release of neurosecretory vesicle-targeted luciferase from neuronal cells. ALTEX 32, 297-306. doi:10.14573/altex. 1503061

Pathe-Neuschäfer-Rube, A., Neuschäfer-Rube, F., Haas, G. et al. (2018). Cell-based reporter release assay to determine the potency of proteolytic bacterial neurotoxins. Toxins (Basel) 10, 360. doi:10.3390/toxins 10090360

Pellett, S., Tepp, W. H., Clancy, C. M. et al. (2007). A neuronal cell-based botulinum neurotoxin assay for highly sensitive and specific detection of neutralizing serum antibodies. FEBS Lett 581, 4803-4808. doi:10.1016/j.febslet.2007.08.078

Pellett, S., Tepp, W. H., Toth, S. I. et al. (2010). Comparison of the primary rat spinal cord cell (RSC) assay and the mouse bioassay for botulinum neurotoxin type A potency determination. J Pharmacol Toxicol Methods 61, 304-310. doi:10.1016/j. vascn.2010.01.003

Pellett, S., Du, Z. W., Pier, C. L. et al. (2011). Sensitive and quantitative detection of botulinum neurotoxin in neurons derived from mouse embryonic stem cells. Biochem Biophys Res Commun 404, 388-392. doi:10.1016/j.bbrc.2010.11.128

Pellett, S., Tepp, W. H., Johnson, E. A. et al. (2017). Assessment of ELISA as endpoint in neuronal cell-based assay for bont detection using hiPSC derived neurons. J Pharmacol Toxicol Methods 88, 1-6. doi:10.1016/j.vascn.2017.04.013

Pirazzini, M., Rossetto, O., Eleopra, R. et al. (2017). Botulinum 
neurotoxins: Biology, pharmacology, and toxicology. Pharmacol Rev 69, 200-235. doi:10.1124/pr.116.012658

Rosen, O., Ozeri, E., Barnea, A. et al. (2016). Development of an innovative in vitro potency assay for anti-botulinum antitoxins. Toxins (Basel) 8, 276. doi:10.3390/toxins8100276

Rusnak, J. M. and Smith, L. A. (2009). Botulinum neurotoxin vaccines: Past history and recent developments. Hum Vaccin 5, 794-805. doi:10.4161/hv.9420

Rust, A., Doran, C., Hart, R. et al. (2017). A cell line for detection of botulinum neurotoxin type B. Front Pharmacol 8, 796. doi:10.3389/fphar.2017.00796

Schiavo, G., Santucci, A., Dasgupta, B. R. et al. (1993). Botulinum neurotoxins serotypes A and E cleave SNAP-25 at distinct cooh-terminal peptide bonds. FEBS Lett 335, 99-103. doi:10.1016/0014-5793(93)80448-4

Schiavo, G., Matteoli, M. and Montecucco, C. (2000). Neurotoxins affecting neuroexocytosis. Physiol Rev 80, 717-766. doi:10.1152/physrev.2000.80.2.717

Smith, L. A. and Rusnak, J. M. (2007). Botulinum neurotoxin vaccines: Past, present, and future. Crit Rev Immunol 27, 303318. doi:10.1615/CritRevImmunol.v27.i4.20

Tannenbaum, J. and Bennett, B. T. (2015). Russell and Burch's 3Rs then and now: The need for clarity in definition and purpose. J Am Assoc Lab Anim Sci 54, 120-132.

Taylor, K., Gericke, C. and Alvarez, L. R. (2019). Botulinum toxin testing on animals is still a Europe-wide issue. ALTEX 36, 81-90. doi:10.14573/altex.1807101

Torgeman, A., Diamant, E., Levin, L. et al. (2017a). An in vitro cell-based potency assay for pharmaceutical type A botulinum antitoxins. Vaccine 35, 7213-7216. doi:10.1016/j. vaccine.2017.11.015

Torgeman, A., Mador, N., Dorozko, M. et al. (2017b). Efficacy of inactivation of viral contaminants in hyperimmune horse plasma against botulinum toxin by low $\mathrm{pH}$ alone and combined with pepsin digestion. Biologicals 48, 24-27. doi:10.1016/j. biologicals.2017.06.003

Torgeman, A., Schwartz, A., Diamant, E. et al. (2018). Studying the differential efficacy of postsymptom antitoxin treatment in type A versus type B botulism using a rabbit spirometry model. Dis Model Mech 11, dmm035089. doi:10.1242/dmm.035089

US FDA - U.S. Food and Drug Administration (2018). Bioanalytical Method Validation - Guidance for Industry. https:// www.Fda.Gov/files/drugs/published/bioanalytical-methodvalidation-guidance-for-industry.pdf

Wang, J., Meng, J., Lawrence, G. W. et al. (2008). Novel chimeras of botulinum neurotoxins $\mathrm{A}$ and $\mathrm{E}$ unveil contributions from the binding, translocation, and protease domains to their functional characteristics. J Biol Chem 283, 16993-17002. doi:10.1074/jbc.M710442200

Whelan, S. M., Elmore, M. J., Bodsworth, N. J. et al. (1992). The complete amino acid sequence of the Clostridium botulinum type-E neurotoxin, derived by nucleotide-sequence analysis of the encoding gene. Eur J Biochem 204, 657-667. doi:10.1111/j.1432-1033.1992.tb16679.x

Wild, E., Bonifas, U., Klimek, J. et al. (2016). In vitro potency determination of botulinum neurotoxin B based on its receptor-binding and proteolytic characteristics. Toxicol In Vitro 34, 97-104. doi:10.1016/j.tiv.2016.03.011

Yadirgi, G., Stickings, P., Rajagopal, S. et al. (2017). Immuno-detection of cleaved SNAP-25 from differentiated mouse embryonic stem cells provides a sensitive assay for determination of botulinum a toxin and antitoxin potency. J Immunol Methods 451, 90-99. doi:10.1016/j.jim.2017.09.007

Zarebski, L. M., Vaughan, K., Sidney, J. et al. (2008). Analysis of epitope information related to Bacillus anthracis and Clostridium botulinum. Expert Rev Vaccines 7, 55-74. doi:10.1586/14760584.7.1.55

\section{Conflict of interest}

The authors declare that they have no conflicts of interest

\section{Acknowledgement}

This work was supported by Israel Institute for Biological Research grant no. SB-190. 